\title{
Los adjetivos en el desarrollo léxico tardío: análisis de narraciones escritas por estudiantes secundarios ${ }^{*}$
}

\author{
Adjectives in late lexical development: analysis of narrations \\ written by secondary students
}

Soledad Aravena $^{* *}$, Riva Quiroga ${ }^{* * *}$

\begin{abstract}
RESUMEN
Se aplicó una tarea de escritura narrativa a 120 estudiantes chilenos de tres niveles secundarios y dos grupos socioeconómicos. En sus textos se analizaron los adjetivos en términos de frecuencia y en cuanto a sus rasgos morfológicos (simples, derivados), sintácticos (función predicativa, modificadora y prenominales, posnominales) y semánticos (calificativos, relacionales). Los resultados muestran que el factor edad/nivel escolar es significativo para todas las medidas analizadas, mientras que el factor socioeconómico solo incide en el uso de adjetivos relacionales. Esto se interpreta en términos de su potencial para referir a las entidades de modo preciso y especializado, rasgos propios del lenguaje de la escuela. Se concluye la gran pertinencia de los adjetivos para estudiar cómo se manifiesta y evoluciona la riqueza léxica durante la adolescencia.
\end{abstract}

\section{SUMMARY}

A narrative writing task was assigned to 120 Chilean students from three secondary levels and two socioeconomic groups. In their texts, adjectives were analyzed in terms of frequency and in terms of their morphological (simple, derived), syntactic (predicative, modifying and pre-nominal, post-nominal) and semantic (qualifying, relational) characteristics. The results show that
Palabras clave: adjetivos, desarrollo, escritura narrativa, grupos socioeconómicos.

Keywords: adjectives, development, narrative writing, socioeconomic groups

* Este estudio ha sido posible gracias al financiamiento del programa Fondecyt (Proyecto 11110191).

** Chilena. Doctora en Lingüística por la Universidad de Barcelona. Profesora del Departamento de Ciencias del Lenguaje, Pontificia Universidad Católica de Chile (PUC). Santiago, Chile.maravena@uc.cl

*** Chilena.Candidata a Doctora en Lingüística, PUC. Santiago, Chile. riva.quiroga@uc.cl 
Los adjetivos en el desarrollo léxico tardío: análisis de narraciones escritas | Aravena y Quiroga

the age / school level factor is significant for all the measures analyzed, while the socioeconomic factor only affects the use of relational adjectives. This is interpreted in terms of its potential to refer to entities in a precise and specialized way, and characteristics of the language of the school. The study concluded that adjectives of study are of great relevance and that lexical richness manifests itself and evolves during adolescence. 


\section{Introducción}

Desde muy temprano, el crecimiento del vocabulario propicia el desarrollo conceptual y cognitivo de los niños (Waxman, 1994) al punto de suministrar la base psicológica para el pensamiento abstracto (Luria, 1995). El conocimiento de las palabras se vincula también con el desarrollo de muchas habilidades complejas, tales como la lectura, la escritura y el rendimiento académico en general (Nagy y Townsend, 2012). Por esto, el dominio creciente de las palabras constituye, junto al de las estructuras sintácticas, el pilar fundamental de la adquisición del lenguaje en los niños pequeños (Clark, 2009) y un aspecto también crucial en los desarrollos tardíos del lenguaje, es decir, los desarrollos del lenguaje que acontecen después de la primera infancia y durante la adolescencia (Nippold, 2016). Para analizar el desarrollo léxico y comprender su impacto, los estudios se han centrado en diversos aspectos, tales como la densidad (Johansson, 2008) y la diversidad léxicas (Malvern et al., 2004), la extensión de las palabras o su complejidad morfológica (Marinellie y Kneile, 2012), su disponibilidad (Callealta y Gallego, 2016), y su grado de sofisticación (Kyle y Crossley, 2016), entre otros rasgos. Como es lógico, el estudio de las palabras de contenido léxico -sustantivos, verbos, adjetivos y algunos adverbios- resulta también central para comprender el desarrollo del vocabulario, puesto que son estas las responsables de la densidad informativa de los mensajes (Ravid y Berman, 2006). En este campo, son numerosas las investigaciones acerca de verbos (Fusté-Herrmann et al., 2006) y sustantivos (Ravid, 2006), ya que conforman categorías imprescindibles para cualquier aproximación evolutiva a los desempeños léxicos.

Los adjetivos, en cambio, tienen una naturaleza menos central que la de verbos y sustantivos. En la gran mayoría de lenguas, de hecho, no gozan de un estatus del todo independiente que pueda compararse con las claras distinciones existentes entre las otras dos principales categorías léxicas (Dixon, 1982). Es debido a esta cierta condición marginal en los estudios lingüísticos tradicionales que los adjetivos también han sido menos atendidos en los trabajos evolutivos respecto del léxico. Sin embargo, en la perspectiva funcional asumida en la presente investigación, se considera que los adjetivos constituyen herramientas importantes para enriquecer el significado de las expresiones lingüísticas y mejorar la coherencia y la cohesión de los textos (Cutillas 
y Tolchinsky, 2017). A diferencia de los sustantivos, que condensan las condiciones suficientes y necesarias para referir a una entidad, los adjetivos son términos generales que pueden aplicarse a múltiples objetos, otorgándoles rasgos extraordinariamente diversos y complejos desde el punto de vista léxico-conceptual (Demonte, 1999). Junto con su enorme riqueza semántica, los adjetivos son además una categoría muy versátil desde el punto de vista sintáctico, en cuanto a sus funciones y posiciones en la oración (McNally y Kennedy, 2008). Así, por su carácter más opcional y su gran complejidad semántica y sintáctica, los adjetivos resultan especialmente pertinentes para estudiar cómo se manifiesta y evoluciona la riqueza léxica en el periodo de los desarrollos tardíos del lenguaje (Ravid y Levie, 2010).

En este marco, el presente estudio se propuso analizar aspectos morfológicos, sintácticos y semánticos de los adjetivos en narraciones escritas por estudiantes de tres niveles escolares y dos grupos socioeconómicos.

\section{El adjetivo: definiciones y alcances para el presente estudio}

En esta investigación se adopta la noción de adjetivo que postula Violeta Demonte en el capítulo que se le dedica a esta categoría en la Gramática descriptiva de la lengua española (1999), entendiéndola como una clase de palabra que modifica al sustantivo y denota propiedades o cualidades del mismo, al mismo tiempo que aporta significados diversos acerca del modo en que se mencionan y relacionan las entidades. El término se utiliza en sentido restrictivo, es decir, como elemento léxico de clase abierta, excluyendo cuantificadores y determinantes e incluyendo, además de los adjetivos léxicos propios (como en "clase aburrida"), los sustantivos adjetivados (como en "operación retorno") y los participios que funcionan como adjetivos (como en "ejercicio terminado") (Millán, 1987; Real Academia Española, RAE, 2010).

En primer lugar, se pretende explorar la dimensión morfológica de los adjetivos, los cuales han sido descritos desde esta perspectiva según una dicotomía simple/complejo, donde lo simple estaría formado por palabras monomorfemáticas (como "gratis") y lo complejo, por palabras plurimorfemáticas (RAE, 2010). En este grupo se incluyen los adjetivos típicamente considerados derivados ("ina- 
propiado"), los compuestos ("agridulce") y las locuciones ("común y corriente"). Se trata de una distinción pertinente para este estudio, porque los adjetivos derivados aparecen con mayor frecuencia entre los estudiantes mayores y, en cambio, implican procesos componenciales demasiado complejos para los niños de menor edad (Marinellie y Kneile, 2012). Su análisis evolutivo es relevante, además, porque el contacto de los niños con palabras derivadas se ha relacionado con el incremento en el volumen de vocabulario y de conocimientos lingüísticos, particularmente semánticos y sintácticos (Anglin, 1993; Tyler y Nagy, 1989).

En el nivel sintáctico, se analizará la función y la posición del adjetivo. En particular, distinguiremos las funciones predicativa $\mathrm{y}$ modificadora ${ }^{l}$ : así, los adjetivos que aquí llamaremos predicativos modifican al sustantivo indirectamente, es decir, mediante un verbo copulativo ("eres veloz") o no copulativo ("huyó despavorida"); en tanto, los que llamaremos modificadores, modifican directamente al sustantivo ("calle estrecha", "lamentable espectáculo"). Algunos estudios han demostrado que la función predicativa es más temprana que la modificadora, puesto que esta última requeriría una integración lógico-semántica de dos etapas, más compleja para los niños pequeños (Nelson, 1976; Ninio, 2004). Además, en términos de la construcción oracional, los modificadores funcionan habitualmente de manera más opcional, mientras que los predicativos resultan más obligatorios, dada la estructura sintáctica que los contiene. Así, una mayor frecuencia de adjetivos modificadores se puede relacionar con construcciones más elaboradas, las que a su vez serían típicas de estudiantes de más edad y niveles escolares superiores. Ello, porque el grupo nominal con un adjetivo en función modificadora puede concentrar una mayor densidad informativa (en el sentido de empaquetar más información) y, a la vez, permite una construcción sintáctica más extensa y eventualmente también más compleja. Ambas - la densidad informativa y la complejidad sintáctica, en particu-

$1 \quad$ Si bien Demonte (1999: §3.1) usa el término "atributo" para referirse al adjetivo en posición inmediata al sustantivo, en este estudio hemos preferido una terminología empleada anteriormente en trabajos que abordan el adjetivo desde una perspectiva aplicada al desarrollo del lenguaje (por ejemplo, Ninio, 2004; Ravid y Levie, 2010; Cutillas y Tolchinsky, 2017). 
lar la mayor complejidad de la frase nominal - han sido relacionadas con la producción típica de estudiantes mayores (Berman y Nir, 2010) y con los usos propios de la escritura académica (Biber, 1988; Parkinson y Musgrave, 2013).

Respecto de la posición, en este trabajo analizaremos adjetivos antepuestos o prenominales y adjetivos pospuestos o posnominales. Como sabemos, algunos adjetivos se sitúan antes del sustantivo, ocupando la posición prenominal, y otros usos adjetivales prefieren la posición posnominal (Bosque y Picallo, 1996). Esta posición no marcada es más frecuente en castellano y, por lo tanto, se presume que será la más habitual en el corpus, y también que será más abundante en los estudiantes de menor edad. Los adjetivos prenominales, en cambio, son más frecuentes en los estudiantes mayores. Ello, por su carácter no especificativo, que no restringe la extensión del sustantivo (como en "niñas rubias" o "libros electrónicos"), sino que más bien lo habilita para destacar o evaluar un rasgo de su significado (como en "hostil recibimiento" o "emocionante discurso") (Demonte, 1999). Así, un adjetivo prenominal tiene un carácter aún más optativo que uno posnominal.

En el nivel semántico, se distinguen habitualmente los adjetivos calificativos, relacionales y modales o adverbiales (Demonte, 1999). En este estudio fueron analizados los tres tipos, pero por razones de espacio y magnitud de los datos obtenidos, solo daremos cuenta de los resultados de calificativos y relacionales (los modales, no obstante, son muy interesantes desde el punto de vista evolutivo y serán objeto de un trabajo posterior). Los calificativos indican cualidades constitutivas de las entidades y que se manifiestan a través de una propiedad única como el color, la textura, la forma, etc. (Demonte, 1999). Por ejemplo, en "día oscuro", la propiedad que se informa es la oscuridad del día. Estos adjetivos, además, aluden a propiedades que siempre pueden ser graduables o medibles y por ello pueden ser modificados a su vez por adverbios (Bosque y Picallo, 1996), como en los ejemplos "(muy) distendida charla", “color (más) opaco" o "conducta (menos) grave". Asimismo, los adjetivos calificativos tienen la capacidad de conformar construcciones binarias de polaridad (opaco/brillante; tranquilo/inquieto) y muy habitualmente pueden ser usados como atributos predicativos (Demonte, 1999). 
Por su parte, los adjetivos relacionales (también llamados clasificadores o categorizadores) adscriben a un conjunto de propiedades que modifican al objeto o entidad, poniéndolo en relación con otras entidades, como en los ejemplos "dato estadístico", “discurso presidencial" o "resolución judicial". Aquí, los adjetivos no expresan una sola propiedad, sino que se refieren a un conjunto de propiedades, las que permiten definir los sustantivos "estadística", "presidente" y “juez" (Bosque, 1993; RAE, 2010). Los adjetivos relacionales deben satisfacer tres rasgos fundamentales que los distinguen de los adjetivos calificativos: no son medibles ni graduables (*capacidad muy respiratoria); no establecen relaciones binarias de polaridad (no tienen antónimos); rechazan (generalmente) ser usados como atributos predicativos (*el discurso fue presidencial) y no aparecen antepuestos. Sin embargo, el contexto puede hacer que un adjetivo relacional (ella estudia arte dramático) se utilice como adjetivo calificativo (su despedida fue muy dramática) y en este caso puede cumplir con todas las condiciones de los adjetivos calificativos (Demonte, 1999).

\section{Metodología}

Selección de la muestra y delimitación del corpus de trabajo

La muestra original consideró a 480 estudiantes, pertenecientes a dos colegios de Santiago - uno subvencionado y otro particular pagadode comunas representativas de dos grupos socioeconómicos (mediobajo y alto, según el Ministerio de Educación de Chile). Ambos colegios son mixtos, imparten los contenidos del currículo nacional y obtienen puntajes Simce (Sistema de Medición de la Calidad de la Educación) cercanos al promedio de su dependencia y comuna. Los estudiantes cursaban los niveles de séptimo básico (SEP), primero medio (PRI) y tercero medio (TER), conformando así un rango etario considerado relevante para observar cambios en los desarrollos tardíos del lenguaje (Nippold, 2016). Además, los niveles inferior y superior pueden informar acerca de conductas de entrada y (próxima) salida de la enseñanza media en el sistema escolar chileno.

Para recoger el corpus, se mostró a los estudiantes un breve video acerca del tema "Internet y las redes sociales" (el más votado por los estudiantes en una encuesta realizada con anterioridad) y luego se les solicitó escribir dos textos - uno narrativo y otro explicativo (que 
no será considerado en este estudio). Excluidos los casos atípicos (es decir, todos los sujetos con rendimiento sobre y bajo las 1,96 unidades de desviación estándar, lo que se conoce como puntaje Z), se seleccionaron de manera aleatoria 120 participantes -20 estudiantes de cada nivel y establecimiento (10 hombres y 10 mujeres)-, cuyas 120 historias personales conforman el corpus definitivo del presente estudio. Se decidió trabajar con 120 participantes tras un análisis de potencia estadística calculado en $\mathrm{G}^{*}$ Power (Faul et al., 2007), que estableció un $N$ muestral mínimo de 108 sujetos. Esta cifra garantizaba óptimas posibilidades de detectar significaciones estadísticas asociadas a un tamaño de efecto cercano al típicamente considerado como mediano $(0,25)$.

\section{Transcripción y segmentación de los textos}

Los 120 textos fueron escaneados, luego transcritos en Word y en CHAT - formato que permite hacer los análisis en CLAN (MacWhinney, 2014)-, siguiendo un riguroso protocolo de transcripción, segmentación y revisión. Para garantizar la consistencia, 40 textos (el $30 \%$ del corpus) fueron transcritos dos veces por distintos investigadores y luego cotejados por un tercero, recogiendo lo señalado en la bibliografía para este tipo de procedimientos (Neuendorf, 2002; Stemler, 2004), y cautelando constantemente la calibración entre transcriptores.

\section{Variables y etiquetado del corpus}

Las variables independientes de este estudio son la edad/nivel escolar y el grupo socioeconómico de los sujetos; las variables dependientes son productividad adjetival y rasgos morfológicos, sintácticos y semánticos de los adjetivos, lo que se traduce en las siguientes medidas: proporción de adjetivos por texto sobre el total de palabras, proporción de adjetivos simples y derivados, proporción de adjetivos en función predicativa y modificadora, proporción de adjetivos prenominales y posnominales y proporción de adjetivos calificativos y relacionales. Los adjetivos fueron etiquetados manualmente, debido a que era necesario distinguir como adjetivos ciertas palabras y expresiones con función adjetiva que no eran reconocidas como tales por el programa, por ejemplo, las locuciones y algunos participios. 


\section{Resultados}

Los resultados generales de esta investigación revelan que los estudiantes mayores utilizan un número y una proporción significativamente mayor de adjetivos en sus historias, como se expone a continuación.

Como se observa en la Tabla 1, la edad/nivel es un factor determinante en el uso de adjetivos, no solo en su frecuencia absoluta (que aumenta, lógicamente, con el número de palabras de los textos), sino también en su frecuencia relativa o proporcional. Los estudiantes menores (séptimo) de ambos establecimientos tienen una proporción de adjetivos cercana al 2,5\%, mientras que los estudiantes mayores (tercero) muestran una proporción que oscila entre el 3,6\% (en el GS medio) y el 4,5\% (en el GS alto). El análisis de varianza muestra que esta diferencia es significativa $(F(1,114)=$ $11,673 ; p=, 001)$ y los análisis posthoc muestran que la significatividad estadística se da entre los niveles de séptimo y primero ( $p=$ ,007) y entre séptimo y tercero $(p=, 001)$, considerando los dos grupos socioeconómicos de manera conjunta. La comparación entre los establecimientos no arroja diferencias significativas y, si miramos lo que ocurre al interior de cada establecimiento, constatamos que la única diferencia significativa se da entre el grupo menor y el mayor en el GS alto $(p=, 001)$.

Tabla 1

Frecuencia absoluta y relativa de adjetivos. Media grupal y desviación estándar (DE)

\begin{tabular}{|c|c|c|c|c|c|c|c|c|c|c|}
\hline & $\begin{array}{l}\text { ppo } \\
\text { ivel } \\
\text { olar }\end{array}$ & $\begin{array}{l}\text { Total } \\
\text { palabras } \\
\text { Media } \\
\text { (DE) }\end{array}$ & Mín. & Máx. & $\begin{array}{l}\text { Total } \\
\text { adjetivos } \\
\text { Media } \\
\text { (DE) }\end{array}$ & Mín. & Máx. & $\begin{array}{l}\text { Proporción* } \\
\text { adjetivos } \\
\text { Media (DE) }\end{array}$ & Mín. & Máx. \\
\hline & Sép. & $232(89,8)$ & 94 & 443 & $5,80(3,9)$ & 0 & 14 & $0,024(0,01)$ & 0 & 0,06 \\
\hline \multirow[t]{3}{*}{ M } & Pri. & $198(98,8)$ & 86 & 349 & $6,45(4,1)$ & 0 & 19 & $0,035(0,02)$ & 0 & 0,07 \\
\hline & Ter. & $295(140,8)$ & 116 & 668 & $10,50(5,9)$ & 3 & 24 & $0,036(0,01)$ & 0,02 & 0,06 \\
\hline & Sép. & $178(42,4)$ & 108 & 251 & $4,60(2,2)$ & 2 & 9 & $0,026(0,01)$ & 0,01 & 0,04 \\
\hline \multirow[t]{2}{*}{ A } & Pri. & $302(106,8)$ & 155 & 584 & $10,60(6,5)$ & 1 & 26 & $0,035(0,02)$ & 0,00 & 0,07 \\
\hline & Ter. & $442(152,5)$ & 166 & 731 & $19,55(8,0)$ & 4 & 35 & $0,045(0,02)$ & 0,01 & 0,09 \\
\hline
\end{tabular}

*Proporción de adjetivos sobre el total de palabras del texto.

Fuente: Elaboración propia. 
A continuación se muestran dos ejemplos que ilustran estos resultados generales. El Ejemplo 1 es un texto de séptimo año básico del GS medio, con 332 palabras y 7 adjetivos (marcados en negrita), es decir, presenta un 2,1\% de adjetivos (proporción inferior a la media de su grupo que es $2,4 \%$ ). Es un texto típicamente infantil cuya escasa complejidad léxica, sintáctica y textual se percibe a simple vista. El Ejemplo 2 corresponde a un texto de tercer año medio del GS alto, con 357 palabras y 31 adjetivos, es decir, presenta una proporción casi cuatro veces mayor que la del texto anterior, de $8 \%$ de adjetivos (superior a la media de su grupo, que es 5\%). Su construcción es evidentemente más madura en todos los planos. Se han seleccionado estos textos porque, a pesar de tener una extensión similar, ilustran de manera muy clara las mayores diferencias en el uso de adjetivos entre ambos niveles escolares.

Ejemplo 1 (332 palabras, 7 adjetivos)

Esta es la historia de un amigo (él me la contó con lujo de detalles)... Un día cualquiera él estaba revisando su Facebook y le llegó una solicitud de amistad de una chica llamada Francisca Gallardo. Él la aceptó de inmediato aunque no la conocía. Luego de aceptarla ella le dijo "hola", a lo cual mi amigo contestó con "hola cómo estás" y ella respondió "bn y tú". Luego de eso mi amigo le dice que se tiene que ir. Él se desconectó. Al día siguiente él tuvo que ir al colegio, para él era como un día cualquiera. A la hora de salir al recreo, él la vio (vio a Francisca Gallardo). Él la reconoció por la foto de perfil de Facebook. Después de este suceso sus amigas lo llamaron. Grande fue su impresión cuando la vio a ella en el grupo de su amiga, se saludaron amistosamente, ella era algo tímida, por lo cual no pudo entablar una conversación con ella. Cuando llegó a su casa mi amigo se conectó a Facebook y ella estaba conectada. Francisca Gallardo le había hablado diciéndole "hola". Él respondió rápidamente diciéndole "hola, cómo ha estado tu tarde". Al día siguiente él se acercó al grupo de su amiga, ella también estaba ahí. Se saludaron nuevamente pero esta vez hablaron un poco más que el día anterior. Cuando llegó a su casa comenzó a hablar con ella a través de Facebook, y hablaron sobre una diversidad de cosas. Luego de un par de días así ella le dice "oye tú me gustas". Cuando llegó al colegio se le acercó a su grupo. Como las amigas de Francisca sabían que a ella le gustaba mi amigo ellas se fueron y los dejaron solos. Al día siguiente sus amigas lo volvieron a hacer. Ellos se comenzaron a hacer muy amigos. Hablaban y se contaban, pasaban todos los recreos juntos. Él un día jueves se decide hacer una pregunta “ ¿quieres pololear conmigo?” Ella felizmente contestó sí. Y es así como una red social pudo unir a dos personas. 
Ejemplo 2 (357 palabras, 31 adjetivos)

Decidí, un día, que no utilizaría el Internet ni computador, y desconecté el cable del que, durante gran parte de mi vida, había dependido. La verdad es que lo hice porque considero que en este planeta existe demasiada gente adicta al mundo cibernético, y quería probarme a mí misma. Sin mucha dificultad lo logré, ya que mi dependencia a esta diabólica invención solo recaía en trabajos escolares. Fue así como estuve alrededor de 4 meses desconectada. Por supuesto que, desde aquél entonces, tuve que destinar más tiempo cuando de investigación se trataba, ya que debía encontrar en la gran biblioteca el libro adecuado que tuviera lo que necesitaba. Fue extraño ya que mi mente experimentó cambios que poco a poco, se hicieron más notorios. Principalmente, mi mente dio origen a un mundo que se podría denominar como puro, sin mayores influencias. Toda esa basura que rebasaba mi mente anteriormente había desaparecido, dejando un espacio vacío que rellené con la más excéntrica y exótica fantasía. Pude ver en esta creación el diáfano reflejo de mi subconsciente. Fue de ese modo como emprendí una interesante travesía hacia mi interior, que me permitió entender, pero en ningún caso compartir, el acelerado mundo exterior. Esta experiencia me permitió entender el funcionamiento y objetivos de las redes cibernéticas, que sutil y disimuladamente bombardean nuestro subconsciente con sus ideologías, manipulándonos de la manera más perversa para conseguir cumplir sus abominables objetivos. Al darme cuenta de este hecho me invadió una gran tristeza por todos aquellos desgraciados que se encontraban indefensos frente a las garras de esta imparable fuerza. Comprendo que es, para algunos, inmensamente difícil tener la suficiente fuerza de voluntad para desarraigarse del internet, pero una vez que lo han logrado entenderán por qué nunca más volví a utilizar este ilegítimo medio para lograr cualquier tipo de objetivo en mi vida. Sé que al estar escribiendo esto esté quebrando el compromiso, tal vez, más importante que hasta ahora tenga, pero es preciso que lo haga, como un mensaje de advertencia y alerta, para todos aquellos desfavorecidos tengan la posibilidad de hacer el intento de evacuar de aquel círculo vicioso en el que muchos están sumidos, y que, tal vez, nunca jamás van a poder salir.

\section{Resultados para el criterio morfológico}

En cuanto a la estructura morfológica, observamos que el factor edad/ nivel nuevamente resulta ser significativo en la elección de adjetivos presumiblemente más complejos, los derivados, tal como se observa en la Tabla 2. 
Tabla 2

Media grupal de adjetivos simples y derivados*, expresados en proporciones sobre el total de palabras del texto

\begin{tabular}{|c|c|c|c|c|c|c|c|}
\hline & & \multicolumn{3}{|c|}{$\begin{array}{l}\text { Proporción de adjetivos } \\
\text { simples }\end{array}$} & \multicolumn{3}{|c|}{$\begin{array}{l}\text { Proporción de adjetivos } \\
\text { derivados }\end{array}$} \\
\hline & & Media(DE) & Mín. & Máx. & Media(DE) & Mín. & Máx. \\
\hline \multirow{3}{*}{ Medio } & Séptimo & $0,017(0,01)$ & 0,00 & 0,06 & $0,006(0,01)$ & 0,00 & 0,03 \\
\hline & Primero & $0,022(0,02)$ & 0,00 & 0,06 & $0,012(0,01)$ & 0,00 & 0,04 \\
\hline & Tercero & $0,024(0,01)$ & 0,01 & 0,04 & $0,011(0,01)$ & 0,00 & 0,03 \\
\hline \multirow{3}{*}{ Alto } & Séptimo & $0,018(0,01)$ & 0,00 & 0,03 & $0,008(0,01)$ & 0,00 & 0,02 \\
\hline & Primero & $0,021(0,01)$ & 0,00 & 0,04 & $0,012(0,01)$ & 0,00 & 0,03 \\
\hline & Tercero & $0,026(0,01)$ & 0,01 & 0,05 & $0,019(0,01)$ & 0,00 & 0,04 \\
\hline
\end{tabular}

*No se reportan resultados de locuciones y complejos, porque su número es muy escaso en todo el corpus.

Fuente: Elaboración propia.

Constatamos que tanto los adjetivos simples como los derivados aumentan a medida que los estudiantes avanzan en su escolaridad, lo que resulta esperable si recordamos que todos los adjetivos aumentan considerablemente con la edad. En particular, los simples experimentan un aumento leve de $1,7 \%$ a $2,4 \%$ en el GS medio y de $1,8 \%$ a $2,6 \%$ en el GS alto, pero significativo $(F(1,114)=4,988 ; p=, 008)$. Más notorio, y también más relevante por el incremento evolutivo de la complejidad, es el aumento de los adjetivos derivados: van de un $0,6 \%$ a $1 \%$ en el GS medio y de $0,8 \%$ a $2 \%$ en el GS alto, diferencia también estadísticamente significativa $(F(1,114)=7,235 ; p=, 001)$. Los análisis posthoc realizados mediante el test de Tukey permiten comprobar que las diferencias se dan específicamente entre los niveles de séptimo año básico y tercer año medio $(p=, 000)$, considerando a los estudiantes de ambos establecimientos de manera conjunta. No se observan diferencias significativas entre los GS, aunque es importante destacar que la distancia entre séptimo año básico y tercer año medio es bastante mayor en el GS alto (lo que se puede observar en las cifras de la tabla anterior).

Los Ejemplos 1 y 2 del apartado anterior también sirven para ilustrar las diferencias respecto de la complejidad morfológica entre los estudiantes menores y los mayores. En el primer texto hay 7 adjetivos, de los cuales 5 son simples ( $71 \%$ del total de adjetivos del texto); los 2 adjetivos restantes (el 29\%) son derivados (anterior, conectada). Este texto tiene una proporción de adjetivos derivados similar a la media 
de su grupo (28\%) y una proporción de adjetivos simples algo mayor que la media de su grupo (65\%). El segundo texto tiene 18 adjetivos simples y 13 derivados (diabólico, escolares, desconectada, adecuado, notorios, excéntrica, interesante, acelerado, abominables, indefensos, imparable, ilegítimo, vicioso), es decir, $42 \%$ de adjetivos derivados, cifra muy similar a la media de su grupo (39\%).

\section{Resultados para el criterio sintáctico: función y posición}

En cuanto a la dimensión sintáctica, y tal como se muestra en la Tabla 3 , los resultados muestran que los adjetivos en función predicativa no cambian mayormente pero, tal como habíamos previsto, sí lo hacen considerablemente los adjetivos con función modificadora y en posición prenominal, la posición marcada y menos habitual.

Tabla 3

Media grupal de predicativos, modificadores y prenominales, expresados en proporciones sobre el total de palabras del texto

\begin{tabular}{cccccccccccc}
\hline & \multicolumn{4}{c}{ Función predicativa } & \multicolumn{4}{c}{ Función modificadora } & \multicolumn{3}{c}{ Posición prenominal } \\
\hline & & Media(DE) & Mín. & Máx. & Media(DE) & Mín. & Máx & Media(DE) & Mín. & Máx. \\
\hline \multirow{3}{*}{ M Sép } & $0,010(0,01)$ & 0,00 & 0,02 & $0,014(0,01)$ & 0,00 & 0,04 & $0,005(0,01)$ & 0,00 & 0,02 \\
\cline { 2 - 11 } & Pri & $0,011(0,01)$ & 0,00 & 0,03 & $0,024(0,02)$ & 0,00 & 0,06 & $0,010(0,01)$ & 0,00 & 0,04 \\
\cline { 2 - 11 } & Ter & $0,012(0,01)$ & 0,00 & 0,03 & $0,023(0,01)$ & 0,00 & 0,04 & $0,011(0,01)$ & 0,00 & 0,03 \\
\hline \multirow{3}{*}{ A } & Sép & $0,011(0,01)$ & 0,00 & 0,04 & $0,015(0,01)$ & 0,00 & 0,04 & $0,007(0,01)$ & 0,00 & 0,03 \\
\cline { 2 - 11 } & Pri & $0,013(0,01)$ & 0,00 & 0,04 & $0,022(0,02)$ & 0,00 & 0,06 & $0,006(0,01)$ & 0,00 & 0,03 \\
\cline { 2 - 10 } & Ter & $0,012(0,01)$ & 0,00 & 0,02 & $0,033(0,01)$ & 0,00 & 0,07 & $0,014(0,01)$ & 0,00 & 0,04 \\
\hline
\end{tabular}

Fuente: Elaboración propia.

Nuevamente constatamos la incidencia del factor edad/nivel, de modo tal que la diferencia en el uso de modificadores entre los menores y mayores es significativa, $(F(1,114)=10,587 ; p=, 001)$ y la distancia entre los menores y mayores es un poco más notoria en el GS alto: aquí los adjetivos modificadores aumentan de 1,5\% a $3 \%$, mientras que en el GS medio lo hacen de $1,4 \%$ a $2,3 \%$. Esto confirma hallazgos anteriores en cuanto a que los niños pequeños usan los adjetivos principalmente con función predicativa (Ninio, 2004) y que a medida que crecen, el uso de la función modificadora se hace más frecuente (Ravid y Levie, 2010). En cuanto a los adjetivos en posición prenominal, también aumentan significativamente a medida que los 
estudiantes avanzan en su escolaridad $(F(1,114)=6,522 ; p=, 000) \mathrm{y}$ a diferencia de lo que ocurre con otras variables, lo hacen de modo similar en ambos establecimientos (de $0,5 \%$ a $1 \%$ en el GS medio y de $0,7 \%$ a $1,4 \%$ en el GS alto). Al analizar las pruebas posthoc, se repite la misma tendencia anterior: tanto para los modificadores como para los prenominales, la diferencia se da entre los niveles de séptimo año básico y tercer año medio, por lo tanto, el cambio se produce después de los 12 años y los estudiantes de 14 y 16 años se comportan de manera similar. Es interesante recordar que los adjetivos prenominales son no especificativos, por lo tanto resultan todavía más opcionales que otros adjetivos de carácter especificativo. Esto proporciona una evidencia más de que durante la adolescencia, concretamente después de los 12 años, los hablantes comienzan a utilizar recursos lingüísticos no indispensables, con finalidades más bien retóricas. Nuevamente, los Ejemplos 1 y 2 permiten ilustrar estas diferencias en el uso de los adjetivos. En el primer texto, 6 de los 7 adjetivos son atributos predicativos (por ejemplo, "grande fue su impresión"). El único adjetivo en función modificadora de este texto ocupa la posición posnominal ("el día anterior"). En el texto del Ejemplo 2, en tanto, los adjetivos modificadores son 23 , lo que equivale al $74 \%$ del total de adjetivos del texto (31). De esos 23, hay 13 en posición prenominal, equivalentes al $57 \%$ del total de modificadores, una cifra muy alta considerando que se trata de la posición menos habitual ("diabólica invención", "excéntrica y exótica fantasía”, etc.). Se evidencia en estos usos el carácter expresivo y valorativo de estos adjetivos, que permiten al hablante posicionarse desde la subjetividad.

\section{Resultados para el criterio semántico}

En cuanto a la dimensión semántica, los resultados de los adjetivos calificativos (el grupo más numeroso) repiten, como es lógico, la tendencia que se viene dando para las demás variables, es decir, su uso aumenta con la edad/nivel. Sin embargo, lo más interesante es el resultado de los adjetivos relacionales, un grupo más reducido que, como veremos, se ve afectado no solo por la edad como todos los demás, sino también por el grupo socioeconómico y por la interacción entre estos dos factores. 
Tabla 4

Media grupal de calificativos y relacionales, expresados en proporciones sobre el total de palabras del texto

\begin{tabular}{llllllll}
\hline \multicolumn{3}{c}{ Calificativo } & & & Relacional \\
\hline & Media(DE) & Mín & Máx & Media(DE) & Mín & Máx \\
\hline \multirow{3}{*}{ M Séptimo } & $0,021(0,01)$ & 0,00 & 0,06 & $0,002(0,01)$ & 0,00 & 0,03 \\
\cline { 2 - 8 } & Primero & $0,031(0,02)$ & 0,00 & 0,07 & $0,004(0,01)$ & 0,00 & 0,02 \\
\cline { 2 - 8 } A & Tercero & $0,031(0,01)$ & 0,02 & 0,06 & $0,003(0,00)$ & 0,00 & 0,02 \\
\hline \multirow{2}{*}{ A Séptimo } & $0,024(0,01)$ & 0,01 & 0,04 & $0,002(0,00)$ & 0,00 & 0,02 \\
\cline { 2 - 8 } & Primero & $0,029(0,01)$ & 0,01 & 0,05 & $0,005(0,01)$ & 0,00 & 0,03 \\
\cline { 2 - 8 } & Tercero & $0,035(0,01)$ & 0,01 & 0,07 & $0,009(0,01)$ & 0,00 & 0,03 \\
\hline
\end{tabular}

Fuente: Elaboración propia.

Se verifica que los adjetivos calificativos aumentan con la edad y lo hacen de manera significativa $(F(1,114)=7,039 ; p=, 001)$ y similar en ambos establecimientos (con una diferencia que va entre $2 \%$ y $3,5 \%$ entre séptimo año básico y tercer año medio). Los adjetivos relacionales son mucho más escasos y se presentan de manera bastante homogénea en todos los cursos del GS medio, aunque con tendencia al alza como hemos visto para otras variables, entre $0,2 \%$ y $0,4 \%$, es decir, entre 2 y 4 adjetivos relacionales cada 1000 palabras. En el GS alto, en cambio, su uso aumenta sostenida y notablemente entre séptimo y tercero medio: de $0,2 \%$ a $1 \%$, o sea, de 2 adjetivos cada 1.000 palabras en séptimo a 1 adjetivo cada 100 palabras en tercero medio. Esto significa que en el GS alto la proporción de los mayores quintuplica a la de los menores. La prueba de varianza revela que, si bien en uno de los colegios la diferencia por edad parece escasa, tomados ambos establecimientos en conjunto el factor edad resulta nuevamente significativo $(F(1,114)=3,970 ; p=, 021)$, particularmente entre los niveles de séptimo y tercero medio, según los resultados de la prueba posthoc $(p=, 015)$. Además, como se ha adelantado, también son significativas las diferencias por GS $(F(1,114)=4,933 ; p=$ ,028) y la interacción entre ambos factores - edad y GS $-(F(2,114)=$ $3,412 ; p=, 036)$, es decir, el patrón evolutivo se produce de forma distinta en los dos establecimientos. En el GS medio, el uso de adjetivos relacionales aumenta moderadamente con la edad, más claramente entre séptimo y primero medio, y luego muestra un leve descenso. En el GS alto, en cambio, se observa un crecimiento sostenido y notable entre séptimo y tercero medio. 
A continuación se presentan dos ejemplos que permiten ilustrar estos resultados. Ambos textos tienen una extensión similar y pertenecen al mismo grupo etario, tercer año medio, pero difieren notablemente en el uso de los adjetivos relacionales, lo cual hace posible ilustrar las diferencias encontradas entre ambos grupos socioeconómicos. El primer texto pertenece al GS medio, tiene 350 palabras y 10 adjetivos, todos calificativos. El segundo texto pertenece al GS alto, tiene 362 palabras y 10 adjetivos relacionales de un total de 18 adjetivos (es decir, $55 \%)$ una proporción muy atípica, incluso considerando las cifras más altas de adjetivos relacionales de toda la muestra.

Ejemplo 3 (350 palabras 10 adjetivos calificativos 0 adjetivo relacional)

Hace un tiempo yo no conocía mucho el asunto de las redes sociales consideraba que era algo aburrido poco interesante o quizás como era pequeña simplemente no me importaba. Luego pasó el tiempo y una amiguita me hizo una cuenta en Facebook lo utilizaba solo para jugar. Cada vez que entraba me divertía bastante pronto comencé a descubrir que este sitio llamado Facebook servía para poder comunicarse con gente por medio de chat y escritura agregué a todos mis amigos del colegio iglesia lugar donde vivo etc. Muy pronto comencé a conversar con cada uno de ellos lo encontraba sinceramente algo fantástico! Como era y es de costumbre yo asistía a la iglesia todos los sábados allí hay muchos jóvenes de mi edad y comparto bastante con quienes más conozco (algunos prácticamente desde bebés). Había un niño que se llamaba Ricardo siempre lo veía pero no intercambiábamos palabras ni mucho menos. Yo me atrevía a hablarle pero un día llegué a mi casa y descubrí que éramos amigos en Facebook (claramente lo conocía de vista quizás por eso lo agregué). Todo comenzó con un hola! Y la conversación del chat se alargaba cada vez más y así día tras día y siempre cuando llegaba el día sábado nos saludábamos y no podíamos charlar libremente. Armamos un grupo y cuando todos salimos de vacaciones de verano comenzamos a salir ya sea al cine a comer a patinar en hielo entre muchas cosas entretenidas. Pero principalmente me preocupé de compartir con mi nuevo amigo Ricardo no sé por qué pero me llamaba la atención. Así transcurrió el tiempo y un día nos juntamos y él se me declaró o sea me dijo que le gustaba ¡no sabía qué hacer! Nunca había pensado o se me pasó por la cabeza que le podría llegar a atraer a él sin embargo me terminó conquistando lo que más me encanta de él es que siempre me saca una sonrisa me canta y es muy sincero conmigo. De una persona que simplemente en un pasado no conocía pasó a ser alguien a quien quiero muchísimo y todo por la iniciativa aburrida de la nueva cuenta en Facebook. 
Ejemplo 4 (362 palabras, 8 adjetivos calificativos y 10 relacionales, subrayados en el texto)

Hace dos años mi profesor de música, mientras estudiábamos audición musical, me hizo escuchar un tema del artista estadounidense John Petrucci. El tema me interesó mucho, al ser un arreglo instrumental de guitarra eléctrica. Quería saber más sobre Petrucci y el grupo de G3 al que pertenecía. En mi casa hice lo que muchos harían en un caso como este, me dirigí hacia mi computador y busqué en Youtube el tema que había escuchado. Luego me fijé en las sugerencias y encontré a los otros dos guitarristas que formaban G3, Joe Satriani y Steve Vai. Después de escuchar algunos de sus temas me di cuenta que G3 era un concierto anual que reunía guitarristas famosos, organizado por Joe Satriani y no un grupo como yo pensaba. En Facebook me hice fan de John Petrucci y G3. Me sugirió otros artistas parecidos como Foofighters. En Wikipedia busqué toda la historia de estos artistas y descubrí que Dove Gral [sic], el líder de Foofighters, había sido el baterista principal del grupo Nirvana antes de su separación. Al volver a Youtube a escuchar todos estos grupos se me ocurrió buscar arreglos de guitarra acústica. Así me encontré con los guitarristas Justin King, Ben Lapps y Eric Mongran. Mi amor por la guitarra no parecía ceder. De vuelta en Facebook me reencontré con la página de G3, y ahí supe que hacían giras por todo el mundo. Mediante blogs y Google supe que no habían venido a Chile desde 2004 más o menos y me puse a pensar si lo harían de nuevo. Busqué por Internet si tenían planeada una gira sudamericana y supe que el 2012 vendrían Joe Satriani, Steve Morse y el que me inició en esta locura, John Petrucci. Hoy yo toco guitarra eléctrica, mi grupo favorito es Foofighters y me mantengo alerta en Facebook para cuando empiecen a vender entradas para el concierto de G3 en Chile. Escucho casi todos los días alguna pieza complicada de guitarra acústica en Youtube e intento aprendérmela mediante tablaturas en Internet. Mi estilo de música preferido es el rock y el instrumental de guitarra. Nunca podría haber descubierto estos grupos de no ser por la Internet.

Como se puede apreciar en el Ejemplo 4, los adjetivos relacionales (entre otros rasgos) contribuyen a dar más especificidad a los términos, al punto que pueden ser considerados como categorías cuasi nominales (Demonte, 1999) y han sido también llamados "pseudoadjetivos" (Bosque, 1993). Esta mayor especificidad hace que se encuentren habitualmente en textos con un carácter más académico, en los que cada una de esas categorías alude a un ámbito semántico acotado y bastante especializado, como en "audición musical”, "guitarra acústica" o "arreglo instrumental". Muchas veces, además, acompañan a sustantivos deverbales o nominalizaciones de tipo resultativo, es decir, sustantivos derivados de verbos, constituyendo expresiones nominalizadas que dan cuenta del resultado de una acción, como en el caso de los sustantivos “audición” y “arreglo”. Ambos rasgos, el léxico altamen- 
te especializado y el uso de nominalizaciones (o expresiones que dan cuenta de procesos de empaquetamiento de información) son propios de hablantes competentes que dominan el lenguaje académico (Snow y Uccelli, 2009). A diferencia de lo que se observaba en el uso de los adjetivos prenominales, más subjetivos y emocionales, estos adjetivos relacionales $-\mathrm{y}$ por lo tanto necesariamente posnominales- dan un significado objetivo y preciso a las entidades. Así, comprobamos que en conjunto (tanto los adjetivos más emocionales como los más objetivos) reflejan una competencia léxica madura y versátil, a la vez que flexible y accesible para el uso (Ravid, 2004).

\section{Conclusiones}

La presente investigación se propuso indagar en los adjetivos -y sus rasgos morfológicos, sintácticos y semánticos- que utilizan estudiantes de tres grupos etarios y dos grupos socioeconómicos en sus narraciones escritas. Las medidas analizadas fueron cinco: proporción de adjetivos por texto, adjetivos derivados, adjetivos en función predicativa y modificadora, adjetivos prenominales y posnominales y adjetivos calificativos y relacionales.

La primera conclusión del estudio - y quizás la más destacablese relaciona con la relevancia del factor edad/nivel escolar, que afecta significativamente todas las medidas analizadas, de tal modo que todos los usos adjetivales se vuelven más sofisticados con la edad. En efecto, a medida que los estudiantes se hacen mayores, utilizan más adjetivos en general; pero también es significativo el aumento de los adjetivos derivados (en la dimensión morfológica), de los que se usan con función modificadora del nombre (en la dimensión sintáctica de función), de los que se ubican antes del nombre (en la dimensión sintáctica de posición), y de los relacionales (en la dimensión semántica). Tal como se ha dicho en la introducción, los adjetivos son formas menos centrales y menos obligatorias que los verbos y sustantivos, lo que se hace evidente cuando se comparan en número con estos últimos. Sin embargo, en tanto unidades de contenido, contribuyen de forma importante a la densidad informativa de los textos. Por estas razones - porque se usan poco, pero aportan mucho- suelen cumplir una función específica en la caracterización de las entidades y tener, por lo tanto, un efecto muy marcado en el sentido de los mensajes. 
En cuanto a la estructura morfológica, los resultados confirman la idea de que el dominio de palabras morfológicamente complejas experimenta un crecimiento sistemático durante los años escolares, gracias al contacto con el lenguaje de la escuela. Los conocimientos morfológicos impactan, a su vez, en el desarrollo mismo del léxico, debido a las estrategias composicionales que permiten a los estudiantes inferir y recordar el significado de nuevas palabras (Anglin, 1993; Sun y Nippold, 2012).

Respecto de la dimensión sintáctica, este estudio también reitera hallazgos anteriores en relación con que la función modificadora del adjetivo es de desarrollo más tardío que la predicativa (Ninio, 2004). Lo anterior se relaciona, como se ha señalado, con el hecho de que esta función favorece una mayor complejidad de la frase nominal. De hecho, diversos estudios han mostrado que muchos de los rasgos típicos del lenguaje académico y particularmente de la escritura académica tales como la concisión, el léxico especializado y el empaquetamiento de información - son observables en la frase nominal, cuya complejidad aumenta sistemáticamente a medida que los estudiantes avanzan en su escolaridad (Biber y Gray, 2016; Parkinson y Musgrave, 2013). Esta creciente complejidad de la frase nominal incluye la incorporación paulatina de diversos tipos de modificadores del nombre, entre los cuales se encuentran los adjetivos pre y posnominales. Mientras que estos últimos, como se ha explicado, sirven típicamente para especificar el sustantivo al que determinan, los adjetivos prenominales se utilizan para desplegarlo y enfatizarlo o atribuirle un contenido emocional, constituyéndose de este modo en un recurso de carácter más retórico (no en el sentido de "accesorio" o menos relevante, sino en el sentido de que, al expresar la subjetividad del hablante, denotan significados extraproposicionales). Son formas frecuentes, por lo tanto, en discursos altamente subjetivos, como textos poéticos o publicitarios y como las historias personales elicitadas en el presente trabajo. Los estudiantes mayores logran utilizarlos pertinentemente en sus historias cuando quieren darles un carácter más afectivo y personal. Este dominio flexible de los recursos lingüísticos según el contexto (género discursivo, modalidad, registro, etc.), propio de una literacidad lingüística competente, solo es posible tras haber alcanzado cierta maduración cognitiva y haber tenido una extensa experiencia con distintas situaciones de comunicación. 
En el ámbito de lo semántico, por último, se obtuvo el resultado acerca de los adjetivos relacionales, que nos conduce a la segunda conclusión del estudio, respecto del efecto del grupo socioeconómico (GS) y de la interacción entre los factores edad y GS. En el GS medio el incremento de estos adjetivos entre séptimo y tercero medio es muy leve, mientras que en el GS alto el incremento es amplio y sostenido: los mayores utilizan cinco veces más de estos adjetivos que los menores en el establecimiento de GS alto.

Es decir, estamos ante un recurso que no solo es de desarrollo tardío y crece con la experiencia (lo que explica el aumento por edad), sino que sobre todo es un rasgo que se beneficia de un mayor contacto con el lenguaje de la escuela. Este se caracteriza por una serie de rasgos léxicos, gramaticales y discursivos, tales como palabras especializadas y de baja frecuencia, estructuras gramaticales que condensan una gran cantidad de información (nominalizaciones y frases nominales extensas y complejas) y conectores, entre otros (Biber y Gray, 2016; Snow y Uccelli, 2009). Es muy interesante constatar que la única medida sensible al factor grupo socioeconómico es la de los adjetivos relacionales, que son justamente típicos de este tipo de lenguaje, con el cual los estudiantes de clases altas están más familiarizados.

En efecto, sabemos que los niños provenientes de las clases medias y altas llegan a primaria con un conjunto de habilidades lingüísticas y experiencias letradas que el sistema valora particularmente y refuerza durante los años escolares (Fang y Park, 2019). Muchos son los factores que favorecen estas condiciones de entrada y han sido estudiados desde diversos ámbitos: los estudios de desarrollo de léxico temprano, por ejemplo, han demostrado que este se ve favorecido por elementos tales como el número de libros que hay en las casas, los años de escolaridad de los padres, particularmente de la madre, y las prácticas lectoras familiares (Hoff, 2003; Snow, 1999). En la misma línea, los estudios de la socialización del lenguaje (LS) indican que las familias de distintos grupos socioeconómicos difieren ampliamente en sus maneras de interactuar con los hijos durante las actividades cotidianas (Pace et al., 2017; Tamis-LeMonda y Bornstein, 2002). En ellas, los padres y madres de clases medias proporcionan a sus hijos oportunidades de adquirir habilidades lingüísticas y discursivas que posteriormente serán reforzadas en la escuela (Blum-Kulka, 2008). Estas habilidades lingüísticas 
facilitan la adquisición de la lectura y el dominio creciente del lenguaje académico, en cuya precisión y densidad informativa contribuyen los adjetivos relacionales y los adjetivos en general, que han sido objeto del presente estudio.

En trabajos posteriores, sería interesante analizar estas medidas en textos orales, para observar eventuales diferencias en el uso de los adjetivos modificadores pre y posnominales, que son rasgos más frecuentes en la escritura (Biber y Gray, 2016). Asimismo, sería necesario también confrontar estos resultados con muestras de otros géneros discursivos, porque probablemente en textos explicativos o informativos, el uso de adjetivos relacionales sea mayor y el de adjetivos de contenido más subjetivo o emocional (adjetivos antepuestos en general) disminuya. Es presumible que los estudiantes mayores que han alcanzado un dominio competente utilicen menos modificadores del nombre en sus conversaciones que en estas narraciones escritas y, asimismo, prefieran más adjetivos posnominales en sus textos expositivos, a diferencia de los que emplean en sus historias. Ello porque, como se ha verificado en el presente estudio, lo que caracteriza un dominio competente del lenguaje (en la adolescencia y la adultez) no es la mayor presencia de tales o cuales recursos lingüísticos, sino el uso flexible que se hace de ellos de acuerdo con las necesidades del contexto y las demandas del género.

\section{Referencias bibliográficas}

Anglin, J. (1993). Vocabulary development: A morphological analysis. Monographs of the Society for Research in Child Development, 58(10), 1-186. https://doi.org/10.2307/1166112

Berman, R. y Nir, B. (2010). The lexicon in writing-speech-differentiation. Developmental perspectives. Written Language \& Literacy, 13(2), 183-205. https://doi.org/10.1075/wll.13.2.01ber

Biber, D. (1988). Variations across speech and writing. Cambridge University Press.

Biber, D. y Gray, B. (2016). Grammatical complexity in academic English. Cambridge University Press.

Blum-Kulka, S. (2008). Language socialization and family dinnertime discourse. En S. May y N. Hornberger (Eds.), Encyclopedia of Language and Education (Vol. 8, pp. 87-100). Springer. 
Bosque, I. (1993). Sobre las diferencias entre los adjetivos relacionales y los calificativos. Revista Argentina de Lingüistica, (9), 9-48.

Bosque, I. y Picallo, C. (1996). Postnominal adjectives in Spanish DPs. Journal of Linguistics, 32(2), 349-385. https://doi. org/10.1017/S0022226700015929

Callealta, F. y Gallego, D. (2016). Medidas de disponibilidad léxica: comparabilidad y normalización. Boletín de Filología, 51(1), 39-92. https://boletinfilologia.uchile.cl/index.php/BDF/article/view/42099/44048

Clark, E. (2009). Lexical meaning. En E. Bavin (Ed.), The Cambridge Handbook of Child Language (pp. 283-300). Cambridge University Press.

Cutillas, L. y Tolchinsky, L. (2017). Use of adjectives in Catalan: A morphological characterization in different genres and modes of production through school-age development. First Language, 37(1) 58-82. https://doi.org/10.1177/0142723716673957

Demonte, V. (1999). El adjetivo: clases y usos. La posición del adjetivo en el sintagma nominal. En I. Bosque y V. Demonte (Eds.), Gramática descriptiva de la lengua española (pp. 129-215). Espasa Calpe.

Dixon, R. (1982). Where have all the adjectives gone? And other Essays in Semantics and Syntax. Mouton De Gruyter.

Fang, Z. y Park, J. (2019). Adolescents' use of academic language in informational writing. Reading and Writing, (33), 97-119. https://doi.org/10.1007/s11145-019-09937-8.

Faul, F., Erdfelder, E., Lang, A., y Buchner, A. (2007). G*Power 3: A flexible statistical power analysis program for the social, behavioral, and biomedical sciences, Behavior Research Methods, (39), 175-191. https://doi.org/10.3758/BF03193146

Fusté-Herrmann, B., Silliman, E., Bahr, R., Fasnacht, K., y Federico, J. (2006). Mental state verb production in the oral narratives of English-and Spanish-speaking preadolescents. Learning Disabilities Research \& Practice, 21(1), 44-60. https://doi. org/10.1111/j.1540-5826.2006.00206.x

Hoff, E. (2003). The specificity of environmental influence: Socioeconomic status affects early vocabulary development via maternal speech. Child Development, 74(5), 1368-1378. https:// doi.org/10.1111/1467-8624.00612 
Johansson, V. (2008). Lexical diversity and lexical density in speech and writing: A developmental perspective, Working Papers, (53), 61-79.

Kyle, K. y Crossley, S. (2016). The relationship between lexical sophistication and independent and source-based writing. Journal of Second Language Writing, (34), 12-24. https://doi. org/10.1016/j.jslw.2016.10.003

Luria, A. (1995). Conciencia y Lenguaje ( $3^{a}$ edición). Visor.

MacWhinney, B. (2014). The CHILDES project: Tools for analyzing talk. https://childes.talkbank.org/

Malvern, D., Richards, B., Chipere, N., y Durán, P. (2004). Lexical diversity and language development. Quantification and Assessment. Palgrave Macmillan.

Marinellie, S. y Kneile, L. (2012). Acquiring knowledge of derived nominals and derived adjectives in context. Language Speech and Hearing Services in Schools, 43(1), 53-65. https://doi. org/10.1044/0161-1461(2011/10-0053).

McNally, L. y Kennedy, C. (2008). Adjectives and adverbs. Syntax, semantics, and discourse. Oxford University Press.

Millán, A. (1987). Sintaxis del adjetivo español: orientación didáctica. Cauce. Revista de Filología y su Didáctica, (10), 201-231.

Nagy, W. y Townsend, D. (2012). Words as tools: Learning academic vocabulary as language acquisition. Reading Research Quarterly, 47(1), 91-108. https://doi.org/10.1002/RRQ.011

Nelson, K. (1976). Some attributes of adjectives used by young children. Cognition, 4(1), 13-30. https://doi.org//10.1016/00100277(76)90008-1

Neuendorf, K. (2002). The content analysis guidebook. Sage.

Ninio, A. (2004). Young children's difficulty with adjectives modifying nouns. Journal of Child Language, (31), 255-285. https://doi. org/10.1017/S0305000904006191

Nippold, M. (2016). Later language development: School-age children, adolescents, and young adults ( ${ }^{\text {th }} \mathrm{Ed}$.). Pro-Ed.

Pace, A., Luo, R., Hirsh-Pasek, K., y Michnick, R. (2017). Identifying pathways between socioeconomic status and language development. Annual Review of Linguistics, 3(1), 285-308. https://doi.org/10.1146/annurev-linguistics-011516-034226 
Parkinson, J. y Musgrave, J. (2013). Development of noun phrase complexity in the writing of English for academic purposes students. Journal of English for Academic Purposes, 14, 48-59. https://doi.org./10.1016/j.jeap.2013.12.001

Ravid, D. (2004). Derivational morphology revisited: Later lexical development in Hebrew. En R. Berman (Ed.), Language development across childhood and adolescence (pp. 53-82). John Benjamin's Publishers.

Ravid, D. (2006). Semantic development in textual contexts during the school years: Noun scale analyses. Journal of Child Language, 33(4), 791-821. https://doi.org/10.1017/s0305000906007586

Ravid, D. y Berman, R. (2006). Information density in the development of spoken and written narratives in English and Hebrew. Discourse Processes, 41(2), 117-149. https://doi.org/10.1207/ s15326950dp4102_2

Ravid, D. y Levie, R. (2010). Adjectives in the development of text production: Lexical, morphological and syntactic analyses. First Language, (30), 27-55. https://doi. org/10.1177/0142723709350529

Real Academia de la Lengua Española, RAE (2010). Nueva gramática de la lengua española. Grupo Editorial Planeta.

Snow, C. (1999). Social perspectives on the emergence of language. En B. MacWhinney (Ed.), The emergence of language (pp. 257276). Erlbaum.

Snow, C. y Uccelli, P. (2009). The challenge of academic language. En D. Olson y N. Torrance (Eds.), The Cambridge Handbook of Literacy (pp. 92-111). Cambridge University Press.

Stemler, S. (2004). A comparison of consensus, consistency, and measurement approaches to estimating inter-rater reliability. Practical Assessment, Research \& Evaluation 9(1). https:// doi.org/10.7275/96jp-xz07

Sun, L. y Nippold, M. (2012). Narrative writing in children and adolescents: Examining the literate lexicon. Language, Speech, and Hearing Services in Schools 43(1), 2-13. https://doi. org/10.1044/0161-1461(2011/10-0099)

Tamis-LeMonda C. y Bornstein, M. (2002). Maternal responsiveness and early language acquisition. Advances in Child Development and Behavior, (29), 89-127. https://doi.org/10.1016/ S0065-2407(02)80052-0 
Tyler, A. y Nagy, W. (1989). The acquisition of English derivational morphology. Journal of Memory and Language, 28(6), 649-667. https://doi.org/10.1016/0749-596X(89)90002-8

Waxman, S. (1994). The development of an appreciation of specific linkages between linguistic and conceptual organization. Lingua, (92), 229-257. https://doi.org/10.1016/00243841(94)90343-3 